\title{
Considering the turbine back-pressure effect of thermal units to optimize the $P Q \_$power generation in power system
}

\author{
- Luu Huu Vinh Quang
}

University of Technology, VNU-HCM

(Manuscript Received on August 12 $2^{\text {nd }}, 2013$, Manuscript Revised September $30^{\text {th }}, 2013$ )

\begin{abstract}
:
A new algorithm of PQ_power optimization is mentioned and some typical numerical examples are presented in this article. The fuel cost characteristics being obtained in form of superposition of some high order polynomial and sinusoidal functions can approximately simulate the turbine back-pressure effect of the generator units at the electrical thermal
\end{abstract}

stations and solve the problem of economic active power dispatch.

A new loss factor formula expressing the network transmission power losses is a second order polynomial function of generator powers containing a square matrix. This loss factor formula is proposed for optimum solution of generator reactive powers in multi-machine power system.

Keywords: Steady-State Optimization; Turbine Back-Pressure Effect; Power System Operation.

\section{INTRODUCTION}

Optimal pq_power generation (OPQG) containing economic p_power generation (EPG) and optimum q_power generation (OQG) is important problem to be solved in the operation and planning of a power system. The objective of the OPQG problem is to determine the optimal combination of pq_power output of all generator unit so as to meet the required load demand at minimum operating cost while satisfying system technical constraints.

The main objective of an economic power dispatch strategy is to determine the optimal operating state of a power system by optimizing a particular objective while satisfying certain specified physical and operating constraints. In

its most general formulation, the economic power dispatch is a nonlinear, nonconvex, large-scale, static optimization problem.

In reality, the turbine fuel cost characteristics can be obtained in form of some convex fracture, this is notably arised from the back-pressure turbine characteristic, in addition, the turbine regulation method also raises some effect to the appearance of turbine fuel cost characteristics, and it may be briefly called the turbine backpressure effect, practically showing as follows [1],[2]

\section{Trang58}




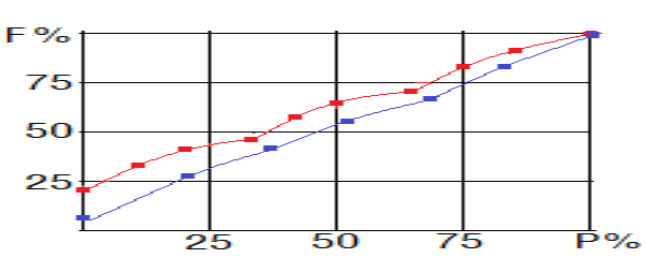

Fig.1 Fuel cost function $F(P)$ in p.u.

In most cases, the fuel cost characteristics of the units rating under $25 \mathrm{MW}$ may be determined experimentally and fit for the $2^{\text {nd }}$ order polynomial function. The fuel cost characteristics of unit rating upper $25 \mathrm{MW}$ may be fit for $n^{\text {th }}$ order polynomial superposing some sinusoidal function to simulate the turbine back-pressure effect of generator on the thermal electrical stations, approximately

$$
C\left(P_{g}\right)=\sum_{m=0}^{n} a_{n-m} P_{g}^{n-m}+b \sin \left(c\left(P_{g}-d\right)\right)
$$

Some typical unit fuel cost characteristics are approximately rated for simulation of turbine back-pressure effect as follows
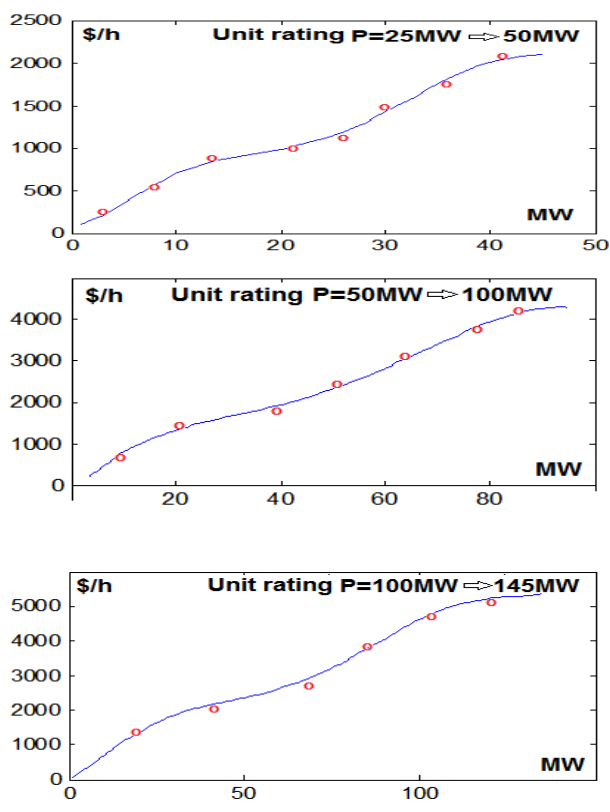

Fig.2 Typical Unit rating fuel cost functions.
This paper is organized as follows 2.Statement of EPG Problem. 3.Statement of OQG problem. 4.Statement of SVC q_power optimization. 5.Numerical examples; 6.Conclusion;

7.References.

\section{STATEMENT OF EPG PROBLEM}

Using the fuel costs (1) to solve the economic p_power dispatch (EPD) problem minimizing the total fuel cost in the whole of electrical power system which consists of many stations. The target function is

$\mathbf{F}=\mathbf{C}\left(P_{g i}\right)+\lambda G\left(P_{g i}, V\right) \rightarrow \min ;$

subject to

$$
\begin{aligned}
& \left(P_{g i}^{-} \leq P_{g i} \leq P_{g_{i}}^{+}\right) ; \\
& i=(1 \ldots N g)
\end{aligned}
$$

where

$C\left(P_{g i}\right)=\sum_{i=1}^{N_{g}}\left(b_{i} \sin \left(c_{i}\left(P_{g i}-d_{i}\right)\right)+\sum_{k=0}^{n} a_{i, n-k} P_{n g i}^{n-k}\right) ;$

$G\left(P_{g i}, V\right)=\Delta P_{P}\left(P_{g i}, V\right)+P_{D}-\sum_{i=1}^{N_{g}} P_{g i} ;$

$\mathrm{N}_{\mathrm{g}}$ is number of generator unit; $\left(\mathrm{i}, \mathrm{m}, \mathrm{r}=1 . . \mathrm{N}_{\mathrm{g}}\right.$ ); $\mathrm{P}_{\mathrm{D}}$ is total MW_power load demand;

$\Delta \mathrm{P}_{\mathrm{P}}\left(\mathrm{P}_{\mathrm{g}}, \mathrm{V}\right)$ is total transmission $\mathrm{MW}$ loss which is taken form in

$$
\Delta P_{P}\left(P_{m g i}, V\right)=\sum_{m=1}^{N_{g}} \sum_{r=1}^{N_{g}} P_{m g i} B_{m r} P_{r g i} ;
$$

MW-loss factors $B_{m r}$ can be found on condition of the $\mathrm{t}^{\text {th }}$ solution of LPF problem [1].

$$
\begin{aligned}
& B_{m r}=\frac{\cos \left(\alpha_{m}-\alpha_{r}\right)}{V_{m} V_{r} \cos \varphi_{m} \cos \varphi_{r}} \\
& \times \sum_{j=1}^{N_{b}} \frac{\left|\sum_{h=1}^{N} C_{j h} J_{h}-C_{j m} J_{\Sigma}\right| \times\left|\sum_{h=1}^{N} C_{j h} J_{h}-C_{j r} J_{\Sigma}\right|}{\left|J_{\Sigma}\right|^{2}} ;
\end{aligned}
$$

where $V_{m}$ is $\mathrm{m}^{\text {th }}$ bus voltage modul; $\alpha_{\mathrm{m}}$ is argument of current output from the $\mathrm{m}^{\text {th }}$ generator; $\cos \varphi_{\mathrm{m}}$ is power factor of $\mathrm{m}^{\text {th }}$ generator; $\mathrm{J}_{\mathrm{h}}$ is $\mathrm{h}^{\text {th }}$ bus current; $\mathrm{J}_{\Sigma}$ is sum of all of bus currents; $\mathrm{C}_{\mathrm{jm}}$ is current distribution factor; 
$\mathrm{N}$ is number of bus $(\mathrm{h}=1 . . \mathrm{N}) ; \mathrm{N}_{\mathrm{b}}$ is number of branch $\left(\mathrm{j}=1 . . \mathrm{N}_{\mathrm{b}}\right)$.

The solution of the problem (2), (3) is iterative calculation of hessian matrix [1], [3], as follows

$$
P_{g}^{(t+1)}=P_{g}^{(t)}-H_{F}^{(t)}\left(\nabla F^{(t)}+\nabla f^{(t)}\right)
$$

where $\mathrm{t}$ is iteration number; $\mathrm{P}_{\mathrm{g}}$ is variable vector of $\mathrm{p} \_$power; $\mathrm{H}_{\mathrm{F}}$ is inversive hessian matrix of target function; $\left(\nabla \mathrm{F}^{(\mathrm{t})}+\nabla \mathrm{f}^{(\mathrm{t})}\right)$ is vector gradient of target function; $\mathrm{f}^{(t)}$ is penalty vector, the $i^{\text {th }}$ element of which is

$f_{i}^{(t)}=\mu\left(\left(P_{g i}^{(t)}-P_{g i}^{+}\right)^{2}+\left(P_{g i}^{(t)}-P_{g i}^{-}\right)^{2}\right) ;$

The iteration process of generator p_power optimization will be converged on condition of $\left(\nabla \mathrm{F}^{(\mathrm{t})}+\nabla \mathrm{f}^{(\mathrm{t})}\right) \rightarrow 0$.

\section{STATEMENT OF OQG PROBLEM}

The OQG problem is to minimize the transmission active power losses, taking into account the steady-state stability margin of every generator in electric power system. The target function is

$$
F_{q}=\sum_{i=1}^{N_{g}} \sum_{j=1}^{N_{g}} Q_{g i} B_{i j}^{*} Q_{g j} \rightarrow m \text { in }
$$

subject to

$$
\begin{aligned}
& Q_{g i}^{-}\left(S_{i}\right) \leq Q_{g i} \leq Q_{g i}^{+}\left(S_{i}\right) ; \\
& V_{i}^{-} \leq V\left(Q_{g}\right)_{i} \leq V_{i}^{+} ; \\
& Q_{D}+\Delta Q_{L}-Q_{C}-\sum_{i=1}^{N_{g}} Q_{g i}=0 ;
\end{aligned}
$$

$\mathrm{Q}_{\mathrm{g}} \mathrm{i}$ is MVAR output from $\mathrm{i}^{\text {th }}$ generator; $\mathrm{i}, \mathrm{j}=1 . . \mathrm{N}_{\mathrm{g}}$;

$\mathrm{S}_{\mathrm{i}}$ is MVA output from $\mathrm{i}^{\text {th }}$ generator;

$\mathrm{Q}_{\mathrm{D}}$ is total MVAR load in power system;

$\Delta \mathrm{Q}_{\mathrm{L}}$ is total transmission MVAR loss in the inductive elements of network; $\mathrm{Q}_{\mathrm{C}}$ is total capacitive reactive power charging of transmission lines.
The solution of the problem (10),(11), (12),(13) is iterative calculation of gradient [1], as follows

$$
Q_{g i}^{(t+1)}=Q_{g i}^{(t)}-\gamma \nabla F_{q}^{(t)}
$$

$\gamma$ is gradient step value;

The $\mathrm{t}^{\text {th }}$ iterative gradient of target function [1] may be determined as follows:

$$
\begin{aligned}
\nabla F_{q}^{(t)} & =2 \sum_{j=1}^{N_{g}} B_{i j}^{*} Q_{g i}^{(t)}+2 \mu_{q} Q_{g i}^{(t)} \\
& -\frac{1}{N_{g}} \sum_{i=1}^{N_{g}}\left(2 \sum_{j=1}^{N_{g}} B_{i j}^{*} Q_{g i}^{(t)}+2 \mu_{q} Q_{g i}^{(t)}\right)
\end{aligned}
$$

$\mu_{\mathrm{q}}$ is penalty factor;

The iteration process of generator q_power optimization will be converged on condition of $\left(\nabla \mathrm{F}^{(\mathrm{t})}{ }_{\mathrm{q}} \rightarrow 10^{-6}\right)$.

\section{STATEMENT OF REACTIVE POWER OPTIMIZATION FOR VAR SUPPORTING DEVICES}

Let's refer to [4],[5]. In this case, the operation conditions requiring a specific steady state at each time interval of load changing in 24 hours, and the test algorithm can reach a purpose which is to solve optimization commitment of device supporting MVAR power, such as TSC, TCR, SVC or synchronous condensers... This proposed mathematical model can be applied to schedule the operation charts of VAR optimization in a power system with multiple voltages level. Here, the problem of optimizing the voltage and q_power in a power system is solved separately for $\mathrm{p} \_$power, i.e. the number of bus of p_power generation in the target network may be generally different from the number of bus of q_power supporting device, and assuming that the bus p_power does not change, it may have been optimized before. The target of VAR optimization problem is established to minimize 
the total cost value [4] consisting of following cost components:

- electrical energy and power losses in the power transmission network;

- installation and operation for var supporting devices;

- depreciation and operation of transfo-LTC;

- q_power generation of power plant;

- optimization of voltage level of power system;

A general form of the objective function of the total cost calculation is written as

$c\left(x_{j}\right)=c_{q b}\left(q_{b j}\right)+c_{d p}\left(x_{j}\right)+c_{m b a}\left(q_{k r}\right)+c_{q g}\left(x_{j}\right)+c_{d u}\left(x_{j}\right) ;$ (16)

where $\mathrm{j}=1,2, \ldots$ number of independent bus in power system; $q_{b} j$ is the controlled MVAR capacity at the bus $(j)$ to meet objective; $c_{q b}\left(q_{b j}\right)$ is the cost of installation and operation for VAR supporting devices; $\mathrm{c}_{\mathrm{dp}}\left(\mathrm{x}_{\mathrm{j}}\right)$ is the cost of electrical energy and power losses in the power transmission network; $\mathrm{c}_{\mathrm{mba}}\left(\mathrm{q}_{\mathrm{kr}}\right)$ is the cost of depreciation and operation of transfo-LTC; $\mathrm{c}_{\mathrm{qg}}\left(\mathrm{x}_{\mathrm{j}}\right)$ is the cost of VAR generation of power plant; $c_{d u}\left(x_{j}\right)$ is the costs optimizing the average voltage level in the power system; $\mathrm{x}_{\mathrm{j}}$ is the controlled variables corresponding to bus (j) to meet objective. The controlled variable $(x)$ is a collective set of the numeric value of voltage module of the power plant busbars and of the transformer station busbar with LTC; or a set of numeric value of VAR capacity of compensation devices located at the bus (i) in power system; or a set of numeric value simulating the transfo LTC at the bus (i) in a power system.

The problem of VAR and voltage optimization is written as follows:

Determine the condition $\mathrm{w}(\mathrm{x}, \mathrm{y}) \rightarrow 0$, such that $\mathrm{c}(\mathrm{x}) \rightarrow \min$ and satisfy the constraints : $\left(\mathrm{v}_{\mathrm{j}}^{-} \leq \mathrm{v}_{\mathrm{j}}\left(\mathrm{q}_{\mathrm{c}}, \mathrm{a}_{\mathrm{j}}\right) \leq \mathrm{v}_{\mathrm{j}}^{+}\right) ; \mathrm{j}=1,2, . .$, total bus number; $\left(\mathrm{q}_{\mathrm{c} j} \geq 0\right)$;

$\left(\mathrm{k}_{\text {min } \mathrm{m}}^{-} \leq \mathrm{k}_{\mathrm{m}}\left(\mathrm{v}_{\mathrm{r}}, \mathrm{a}_{\mathrm{r}}\right) \leq \mathrm{k}_{\text {max }}^{+}\right) ; \mathrm{m}=1,2, \ldots .$, total transformer number;

$\left(\mathrm{q}_{\mathrm{g} i}^{-} \leq \mathrm{q}_{\mathrm{g}}\left(\mathrm{v}_{\mathrm{i}}, \mathrm{a}_{\mathrm{i}}\right) \leq \mathrm{q}_{\mathrm{gi}}^{+}\right) ; \quad \mathrm{i}=1,2, . .$, total PV bus number;

where: $q_{b j}$ is the VAR capacity to be supported at the bus $(j) ;\left(q_{b}, a_{i}\right)$ is the voltage at the $j^{\text {th }}$ bus; $\mathrm{q}_{\mathrm{gi}}\left(\mathrm{v}_{\mathrm{i}}, \mathrm{a}_{\mathrm{i}}\right)$ is the generated VAR capacity of the $i^{\text {th }}$ power plant; $a_{i}$ is the $i_{-}$th element of the eigenimage vector A simulating a certain steady state structure of power system; $\mathrm{k}_{\mathrm{m}}$ is the numeric value corresponds to one simulated ratio of the LTC of $\mathrm{m}$-th transformer; $w(x, y)$ is the vector balance indicating a certain technical condition of steady state of power system; $\mathrm{x}$ is the vector controlled to meet objectives (the $\mathrm{v}_{\mathrm{g}}, \mathrm{q}_{\mathrm{b}}$ and the $\mathrm{k}_{\mathrm{mba}}$ ); $\mathrm{y}$ is the non-controlled vector.

In reality, we can apply some specific factors or choose some parameters depending on concrete conditions of q_power optimization to take account of total cost function $\mathrm{c}\left(\mathrm{x}_{\mathrm{j}}\right)$ or of just one component function.

In this article the component function $\mathrm{c}_{\mathrm{dp}}\left(\mathrm{x}_{\mathrm{j}}\right)$ is used to make the target function of q_power optimization problem. The statement of q_power optimization of VAR supporting device is to determine $c_{d p}\left(q_{b j}\right) \rightarrow \min$. Then, this pq_power optimization problem may be solved with multitarget function by applying the method of optimum co-ordination, i.e. the main function (2) must be satisfied (10) under condition of $\mathrm{c}_{\mathrm{dp}}\left(\mathrm{q}_{\mathrm{bj}}\right) \rightarrow \min$.

\section{NUMERICAL EXAMPLE}

Let's survey the optimum condition operartion of a 68-bus power system consisting of 4 thermal stations with 15 generation units and of 5 SVC stations. Basic power is 100MVA. The linedata is given in p.u. in table 1 as follows: 
Table 1. Linedata

\begin{tabular}{|c|c|c|c|c|}
\hline Bus (i) & Bus (j) & $\mathrm{R}(\mathrm{pu})$ & $\mathrm{X}(\mathrm{pu})$ & $\mathrm{B} / 2(\mathrm{pu})$ \\
\hline 49 & 50 & 0.055207202 & 0.086838638 & 0.019818687 \\
\hline 49 & 51 & 0.033958779 & 0.096990529 & 0.024738886 \\
\hline 49 & 52 & 0.024158946 & 0.069000979 & 0.01759974 \\
\hline 49 & 22 & 0.002620277 & 0.064573756 & 0 \\
\hline 49 & 45 & 0.000870248 & 0.039256198 & 0 \\
\hline 50 & 23 & 0.010239233 & 0.20865162 & 0 \\
\hline 51 & 52 & 0.047584215 & 0.135906483 & 0.008666262 \\
\hline 51 & 24 & 0.010239233 & 0.20865162 & 0 \\
\hline 52 & 25 & 0.010239233 & 0.20865162 & 0 \\
\hline 53 & 54 & 0.079699126 & 0.125363417 & 0.007152746 \\
\hline 53 & 26 & 0.007538333 & 0.162367539 & 0 \\
\hline 53 & 43 & 0.028823037 & 0.082322209 & 0.020997517 \\
\hline 54 & 27 & 0.010239233 & 0.20865162 & 0 \\
\hline 54 & 43 & 0.070558795 & 0.171287676 & 0.010596018 \\
\hline 55 & 56 & 0.035405171 & 0.085949163 & 0.021267541 \\
\hline 55 & 28 & 0.010239233 & 0.20865162 & 0 \\
\hline 56 & 58 & 0.025783517 & 0.073640957 & 0.018783217 \\
\hline 56 & 29 & 0.010239233 & 0.20865162 & 0 \\
\hline 57 & 58 & 0.044083527 & 0.107016632 & 0.02648056 \\
\hline 57 & 30 & 0.010239233 & 0.20865162 & 0 \\
\hline 58 & 16 & 0.139724411 & 0.2197807 & 0.012539811 \\
\hline 58 & 31 & 0.005804919 & 0.129894031 & 0 \\
\hline 58 & 47 & 0.000870248 & 0.039256198 & 0 \\
\hline 59 & 32 & 0.01765663 & 0.323615302 & 0 \\
\hline 59 & 43 & 0.072803366 & 0.070299545 & 0.015033814 \\
\hline 60 & 61 & 0.040505438 & 0.051240957 & 0.011359432 \\
\hline 60 & 33 & 0.01765663 & 0.323615302 & 0 \\
\hline 61 & 62 & 0.042158227 & 0.066313143 & 0.015134293 \\
\hline 61 & 34 & 0.01765663 & 0.323615302 & 0 \\
\hline 62 & 15 & 0.050188366 & 0.078944217 & 0.018016997 \\
\hline 62 & 35 & 0.01765663 & 0.323615302 & 0 \\
\hline 62 & 46 & 0.001539669 & 0.062809917 & 0 \\
\hline 15 & 16 & 0.069460698 & 0.109258798 & 0.006233872 \\
\hline
\end{tabular}

\section{Trang 2}


TAP̈ CH PHAIUTTREÅIKHSON TAR̈ 16, SOÁ3- 20B

\begin{tabular}{|c|c|c|c|c|}
\hline 15 & 36 & 0.01765663 & 0.323615302 & 0 \\
\hline 16 & 37 & 0.01765663 & 0.323615302 & 0 \\
\hline 17 & 18 & 0.056311347 & 0.088575411 & 0.020215083 \\
\hline 17 & 38 & 0.010239233 & 0.20865162 & 0 \\
\hline 18 & 19 & 0.035279399 & 0.085643839 & 0.021191988 \\
\hline 18 & 39 & 0.010239233 & 0.20865162 & 0 \\
\hline 19 & 20 & 0.021884545 & 0.053126659 & 0.013145827 \\
\hline 19 & 40 & 0.010239233 & 0.20865162 & 0 \\
\hline 19 & 48 & 0.000870248 & 0.039256198 & 0 \\
\hline 20 & 21 & 0.045571037 & 0.071681349 & 0.016359442 \\
\hline 20 & 41 & 0.005804919 & 0.129894031 & 0 \\
\hline 21 & 42 & 0.010239233 & 0.20865162 & 0 \\
\hline 43 & 44 & 0.001539669 & 0.062809917 & 0 \\
\hline 44 & 45 & 0.008976942 & 0.04710124 & 0.2668292 \\
\hline 44 & 46 & 0.007107025 & 0.031283058 & 0.17265248 \\
\hline 45 & 47 & 0.01078438 & 0.056584711 & 0.3205532 \\
\hline 47 & 48 & 0.014380847 & 0.06330031 & 0.349357008 \\
\hline 44 & 1 & 0.002066116 & 0.20661157 & 0 \\
\hline 44 & 2 & 0.008264463 & 0.20661157 & 0 \\
\hline 44 & 3 & 0.008264463 & 0.20661157 & 0 \\
\hline 44 & 4 & 0.008264463 & 0.20661157 & 0 \\
\hline 45 & 5 & 0.008264463 & 0.20661157 & 0 \\
\hline 45 & 6 & 0.008264463 & 0.20661157 & 0 \\
\hline 45 & 7 & 0.008264463 & 0.20661157 & 0 \\
\hline 45 & 8 & 0.008264463 & 0.20661157 & 0 \\
\hline 47 & 9 & 0.005454545 & 0.150413223 & 0 \\
\hline 47 & 10 & 0.005454545 & 0.150413223 & 0 \\
\hline 47 & 11 & 0.005454545 & 0.150413223 & 0 \\
\hline 48 & 12 & 0.008264463 & 0.20661157 & 0 \\
\hline 48 & 13 & 0.008264463 & 0.20661157 & 0 \\
\hline 48 & 14 & 0.008264463 & 0.20661157 & 0 \\
\hline 48 & 68 & 0.008264463 & 0.20661157 & 0 \\
\hline 19 & 63 & 0.00072562 & 0.070247934 & 0 \\
\hline 43 & 64 & 0.001283471 & 0.103305785 & 0 \\
\hline 49 & 65 & 0.00072562 & 0.070247934 & 0 \\
\hline
\end{tabular}




\begin{tabular}{|l|l|l|l|l|}
\hline 58 & 66 & 0.00072562 & 0.070247934 & 0 \\
\hline 62 & 67 & 0.001283471 & 0.103305785 & 0 \\
\hline
\end{tabular}

Let's investigate the case of loud load. The busdata of initial operation condition of power system is given in tables $2 \mathrm{a}, 2 \mathrm{~b}$ and $2 \mathrm{c}$ as follows:

Table 2a. SVC-data

\begin{tabular}{|c|c|c|}
\hline Bus (i) & $\begin{array}{c}\text { Max-MVAR } \\
\text { of TCR }\end{array}$ & $\begin{array}{c}\text { Max-MVAR } \\
\text { of TSC }\end{array}$ \\
\hline 63 & -20 & 20 \\
\hline 64 & -30 & 20 \\
\hline 65 & -40 & 30 \\
\hline 66 & -50 & 40 \\
\hline 67 & -30 & 20 \\
\hline
\end{tabular}

Table 2b. Loud Load-data

\begin{tabular}{|c|c|c|c|}
\hline $\begin{array}{c}\text { Bus } \\
\text { (i) }\end{array}$ & $\begin{array}{c}\text { Load } \\
\text { MW }\end{array}$ & $\begin{array}{c}\text { Load } \\
\text { MVAR }\end{array}$ & $\begin{array}{c}\text { Fixed } \\
\text { Capacitor } \\
\text { MVAR }\end{array}$ \\
\hline 49 & 0.14 & 0.96 & 0 \\
\hline 50 & 0.06 & 0.4 & 0 \\
\hline 51 & 0.06 & 0.4 & 0 \\
\hline 52 & 0.06 & 0.4 & 0 \\
\hline 53 & 0.07 & 0.48 & 0 \\
\hline 54 & 0.06 & 0.4 & 0 \\
\hline 55 & 0.06 & 0.4 & 0 \\
\hline 56 & 0.06 & 0.4 & 0 \\
\hline 57 & 0.06 & 0.4 & 0 \\
\hline 58 & 0.08 & 0.56 & 0 \\
\hline 59 & 0.04 & 0.272 & 0 \\
\hline 60 & 0.04 & 0.272 & 0 \\
\hline 61 & 0.04 & 0.272 & 0 \\
\hline 62 & 0.04 & 0.272 & 0 \\
\hline
\end{tabular}

\begin{tabular}{|c|c|c|c|}
\hline 15 & 0.04 & 0.272 & 0 \\
\hline 16 & 0.04 & 0.272 & 0 \\
\hline 17 & 0.06 & 0.4 & 0 \\
\hline 18 & 0.06 & 0.4 & 0 \\
\hline 19 & 0.06 & 0.4 & 0 \\
\hline 20 & 0.08 & 0.56 & 0 \\
\hline 21 & 0.06 & 0.4 & 0 \\
\hline 22 & 77.5 & 58 & 20 \\
\hline 23 & 27.5 & 18.12 & 7 \\
\hline 24 & 35 & 29.47 & 12 \\
\hline 25 & 31 & 24.63 & 10 \\
\hline 26 & 35 & 27.54 & 11 \\
\hline 27 & 25 & 19.68 & 8 \\
\hline 28 & 23 & 18.17 & 7 \\
\hline 29 & 27 & 18.26 & 7 \\
\hline 30 & 31 & 20.96 & 8 \\
\hline 31 & 50 & 33.8 & 15 \\
\hline 32 & 15 & 11.61 & 3 \\
\hline 33 & 17 & 11.2 & 4 \\
\hline 34 & 17 & 14.31 & 6 \\
\hline 35 & 17 & 13.5 & 5 \\
\hline 36 & 17 & 16.47 & 7 \\
\hline 37 & 25 & 20.85 & 9 \\
\hline 38 & 27 & 26.92 & 12 \\
\hline 39 & 21 & 20.36 & 8 \\
\hline 40 & 39 & 30 & 15 \\
\hline 41 & 18 & 17.32 & 7 \\
\hline 42 & 17 & 13.38 & 6 \\
\hline 43 & 0.15 & 1 & 0 \\
\hline 44 & 0.2 & 1.6 & 0 \\
\hline 45 & 0.2 & 1.6 & 0 \\
\hline 46 & 0.15 & 1 & 0 \\
\hline
\end{tabular}

\section{Trang 64}




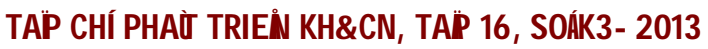

\begin{tabular}{|l|l|l|l|}
47 & 0.2 & 1.6 & 0 \\
\hline 48 & 0.2 & 1.6 & 0 \\
\hline
\end{tabular}

Table 2c. Generator-data

\begin{tabular}{|c|c|c|}
\hline Bus (i) & $\begin{array}{c}\text { Initial Generator } \\
\text { MW }\end{array}$ & $\begin{array}{c}\text { Initial Bus Voltage } \\
\text { (p.u.) }\end{array}$ \\
\hline 1 & 40 & 1.08 \\
\hline 2 & 40 & 1.08 \\
\hline 3 & 40 & 1.08 \\
\hline 4 & 40 & 1.08 \\
\hline 5 & 40 & 1.08 \\
\hline 6 & 40 & 1.08 \\
\hline 7 & 40 & 1.08 \\
\hline 8 & 40 & 1.08 \\
\hline 9 & 40 & 1.08 \\
\hline 10 & 40 & 1.08 \\
\hline 11 & 40 & 1.08 \\
\hline 12 & 40 & 1.08 \\
\hline 13 & 40 & 1.08 \\
\hline 14 & 40 & 1.08 \\
\hline 68 & Pilot-Slack & 1.08 \\
\hline
\end{tabular}

The fuel cost characteristics of thermal unit are given in form of a third order polynomial adding a sinusoidal function of p_power generation:

$C\left(P_{g}\right)=a_{3} P_{g}^{3}+a_{2} P_{g}^{2}+a_{1} P_{g}+a_{0}+b \sin \left(c\left(P_{g}-d\right)\right)$

and the specific data of which is given in table 3 with suitable coefficients as follows:

Table 3. Characteristic coefficients

\begin{tabular}{|c|c|c|c|c|c|c|c|}
\hline Bus & $\mathrm{a}_{3}$ & $\mathrm{a}_{2}$ & $\mathrm{a}_{1}$ & $\mathrm{a}_{\mathrm{o}}$ & $\mathrm{b}$ & $\mathrm{c}$ & $\mathrm{d}$ \\
\hline 1 & 0.0002 & 0.304 & 40.41 & 324 & 270 & 0.126 & 14 \\
\hline 2 & 0.00021 & 0.3039 & 40.45 & 325 & 270 & 0.126 & 14 \\
\hline 3 & 0.00019 & 0.3038 & 40.46 & 323 & 270 & 0.126 & 14 \\
\hline
\end{tabular}

\begin{tabular}{|c|c|c|c|c|c|c|c|}
\hline 4 & 0.0002 & 0.3039 & 40.45 & 324 & 270 & 0.126 & 14 \\
\hline 5 & 0.00019 & 0.3037 & 40.44 & 323 & 270 & 0.126 & 14 \\
\hline 6 & 0.00021 & 0.304 & 40.45 & 324 & 270 & 0.126 & 14 \\
\hline 7 & 0.0002 & 0.3038 & 40.5 & 325 & 270 & 0.126 & 14 \\
\hline 8 & 0.00019 & 0.3039 & 40.5 & 323 & 270 & 0.126 & 14 \\
\hline 9 & 0.00017 & 0.3017 & 39.19 & 303 & 267 & 0.126 & 14 \\
\hline 10 & 0.00017 & 0.3021 & 39.2 & 301 & 267 & 0.126 & 14 \\
\hline 11 & 0.00017 & 0.3019 & 39.18 & 302 & 267 & 0.126 & 14 \\
\hline 12 & 0.00019 & 0.303 & 40.51 & 321 & 270 & 0.126 & 14 \\
\hline 13 & 0.0002 & 0.304 & 40.49 & 322 & 270 & 0.126 & 14 \\
\hline 14 & 0.0002 & 0.303 & 40.49 & 323 & 270 & 0.126 & 14 \\
\hline 68 & 0.00019 & 0.304 & 40.5 & 322 & 270 & 0.126 & 14 \\
\hline
\end{tabular}

Generation limit data is given in table 4 as follows:

Table 4. Generation limit data

\begin{tabular}{|c|c|c|c|}
\hline Bus (i) & $\mathrm{P}_{\min }(\mathrm{MW})$ & $\mathrm{P}_{\max }(\mathrm{MW})$ & $\mathrm{S}_{\text {nominal }}(\mathrm{MVA})$ \\
\hline 1 & 1 & 60 & 62 \\
\hline 2 & 1 & 60 & 62 \\
\hline 3 & 1 & 60 & 62 \\
\hline 4 & 1 & 60 & 62 \\
\hline 5 & 1 & 60 & 62 \\
\hline 6 & 1 & 60 & 62 \\
\hline 7 & 1 & 60 & 62 \\
\hline 8 & 1 & 60 & 62 \\
\hline 9 & 1 & 72 & 75 \\
\hline 10 & 1 & 72 & 75 \\
\hline 11 & 1 & 72 & 75 \\
\hline 12 & 1 & 60 & 62 \\
\hline 13 & 1 & 60 & 62 \\
\hline 14 & 1 & 60 & 62 \\
\hline 68 & 1 & 60 & 62 \\
\hline
\end{tabular}

The pilot-slack bus is $68^{\text {th }}$ and voltage of which is 1.08 p.u.

Typical results are shown in table 5 by comparing the initial powers with the optimum power as follows 
Table 5. Comparison of generation

\begin{tabular}{|r|l|c|c|c|c|}
\hline $\begin{array}{r}\text { Bus } \\
\text { (i) }\end{array}$ & \multirow{2}{*}{$\begin{array}{c}\text { Bus } \\
\text { Code }\end{array}$} & \multicolumn{2}{|c|}{$\begin{array}{c}\text { Initial } \\
\text { Generation }\end{array}$} & \multicolumn{2}{c|}{$\begin{array}{c}\text { Optimum } \\
\text { Generation }\end{array}$} \\
\cline { 3 - 6 } & & MW & MVAR & MW & MVAR \\
\hline 1 & Generator & 40 & 8.7020 & 40.48866 & 0.41239 \\
\hline 2 & Generator & 40 & 7.4868 & 40.31214 & 0.40703 \\
\hline 3 & Generator & 40 & 7.4868 & 40.37952 & 0.40702 \\
\hline 4 & Generator & 40 & 7.4868 & 40.34666 & 0.40701 \\
\hline 5 & Generator & 40 & 8.66521 & 40.3981 & 0.40728 \\
\hline 6 & Generator & 40 & 8.66521 & 40.30547 & 0.4073 \\
\hline 7 & Generator & 40 & 8.66521 & 40.31607 & 0.40733 \\
\hline 8 & Generator & 40 & 8.66521 & 40.34492 & 0.40732 \\
\hline 9 & Generator & 40 & 8.45011 & 41.1155 & 0.40823 \\
\hline 10 & Generator & 40 & 8.45011 & 41.09443 & 0.40826 \\
\hline 11 & Generator & 40 & 8.45011 & 41.11474 & 0.40821 \\
\hline 12 & Generator & 40 & 7.40968 & 40.1322 & 0.39782 \\
\hline 13 & Generator & 40 & 7.40968 & 40.04765 & 0.39783 \\
\hline 14 & Generator & 40 & 7.40968 & 40.11039 & 0.39784 \\
\hline 68 & Generator & 47.38853 & 7.69065 & 40.08194 & -0.47976 \\
\hline 63 & SVC & 0 & 0 & 0 & 19.96 \\
\hline 64 & SVC & 0 & 0 & 0 & 6.87161 \\
\hline 65 & SVC & 0 & 0 & 0 & 26.46949 \\
\hline 66 & SVC & 0 & 0 & 0 & 33.18359 \\
\hline 67 & SVC & 0 & 0 & 0 & 8.9178 \\
\hline $\begin{array}{r}\text { Initial Fuel Cost } \\
\text { Sptimum Fuel Cost }\end{array}$ & $36047.61 \$ / \mathrm{h}$ & & \\
\hline
\end{tabular}

The bus voltages are compered in table 6 as follows

Table 6. Voltage comparison

\begin{tabular}{|c|c|c|}
\hline Bus (i) & $\begin{array}{c}\text { Initial Voltage } \\
\text { (p.u.) }\end{array}$ & $\begin{array}{c}\text { Optimum Voltage } \\
\text { (p.u.) }\end{array}$ \\
\hline 1 & 1.08 & 1.0755 \\
\hline 2 & 1.08 & 1.0778 \\
\hline
\end{tabular}

\begin{tabular}{|c|c|c|}
\hline 3 & 1.08 & 1.0778 \\
\hline 4 & 1.08 & 1.0778 \\
\hline 5 & 1.08 & 1.0786 \\
\hline 6 & 1.08 & 1.0786 \\
\hline 7 & 1.08 & 1.0786 \\
\hline 8 & 1.08 & 1.0786 \\
\hline 9 & 1.08 & 1.0872 \\
\hline 10 & 1.08 & 1.0872 \\
\hline 11 & 1.08 & 1.0872 \\
\hline 12 & 1.08 & 1.0817 \\
\hline 13 & 1.08 & 1.0817 \\
\hline 14 & 1.08 & 1.0817 \\
\hline 15 & 1.0065 & 1.032 \\
\hline 16 & 0.9966 & 1.0248 \\
\hline 17 & 0.9698 & 0.9957 \\
\hline 18 & 1.0000 & 1.025 \\
\hline 19 & 1.0405 & 1.064 \\
\hline 20 & 1.0249 & 1.0489 \\
\hline 21 & 1.0123 & 1.0367 \\
\hline 22 & 0.9995 & 1.0259 \\
\hline 23 & 0.9735 & 1.0009 \\
\hline 24 & 0.9567 & 0.9848 \\
\hline 25 & 0.9697 & 0.9973 \\
\hline 26 & 0.9837 & 1.0012 \\
\hline 27 & 0.981 & 0.9985 \\
\hline 28 & 0.967 & 1.0014 \\
\hline 29 & 0.9843 & 1.0179 \\
\hline 30 & 0.9804 & 1.0141 \\
\hline 31 & 1.0121 & 1.0443 \\
\hline 32 & 0.9937 & 1.0108 \\
\hline 33 & 0.965 & 0.9899 \\
\hline 34 & 0.9725 & 0.9971 \\
\hline 35 & 0.9971 & 1.0209 \\
\hline 36 & 0.9704 & 0.9969 \\
\hline 37 & 0.948 & 0.9779 \\
\hline 38 & 0.9316 & 0.9587 \\
\hline
\end{tabular}

\section{Trang 66}


TAP̈ CH PHAUITTREÅIKHSON TAR̈ 16, SOÁ3- 20B

\begin{tabular}{|c|c|c|}
\hline 39 & 0.9703 & 0.996 \\
\hline 40 & 1.0023 & 1.0268 \\
\hline 41 & 1.0103 & 1.0347 \\
\hline 42 & 0.9945 & 1.0193 \\
\hline 43 & 1.0419 & 1.0581 \\
\hline 44 & 1.0653 & 1.0767 \\
\hline 45 & 1.0631 & 1.0775 \\
\hline 46 & 1.0533 & 1.0687 \\
\hline 47 & 1.0676 & 1.0861 \\
\hline 48 & 1.0655 & 1.0806 \\
\hline 49 & 1.0272 & 1.0529 \\
\hline 50 & 1.0019 & 1.0284 \\
\hline 51 & 1.0013 & 1.028 \\
\hline 52 & 1.0066 & 1.033 \\
\hline 53 & 1.0153 & 1.0321 \\
\hline 54 & 1.0098 & 1.0267 \\
\hline 55 & 0.9947 & 1.0281 \\
\hline 56 & 1.0125 & 1.0451 \\
\hline 57 & 1.0133 & 1.0457 \\
\hline 58 & 1.041 & 1.0722 \\
\hline 59 & 1.0255 & 1.042 \\
\hline 60 & 0.9939 & 1.0179 \\
\hline 61 & 1.0047 & 1.0285 \\
\hline 62 & 1.0291 & 1.052 \\
\hline 63 & 1.0405 & 1.077 \\
\hline 64 & 1.0419 & 1.0647 \\
\hline 65 & 1.0272 & 1.0703 \\
\hline 66 & 1.041 & 1.0936 \\
\hline 67 & 1.0291 & 1.0607 \\
\hline 68 & 1.08 & 1.08 \\
\hline
\end{tabular}

Let's investigate the case of slight load.

In this case, the SVC-data is also refered to the table 2a. The busdata of initial operation condition of power system is given in table $7 \mathrm{a}$ and $7 \mathrm{~b}$ as follows:

Table 7a. Slight Load-data

\begin{tabular}{|c|c|c|c|}
\hline Bus (i) & Load MW & Load MVAR & $\begin{array}{c}\text { Fixed } \\
\text { Capacitor } \\
\text { MVAR }\end{array}$ \\
\hline 15 & 0.042 & 0.272 & 0 \\
\hline 16 & 0.042 & 0.272 & 0 \\
\hline 17 & 0.058 & 0.4 & 0 \\
\hline 18 & 0.058 & 0.4 & 0 \\
\hline 19 & 0.058 & 0.4 & 0 \\
\hline 20 & 0.084 & 0.56 & 0 \\
\hline 21 & 0.058 & 0.4 & 0 \\
\hline 22 & 31 & 34.88 & 20 \\
\hline 23 & 12.65 & 7.98 & 7 \\
\hline 24 & 19.6 & 21.7 & 12 \\
\hline 25 & 22.01 & 16.74 & 10 \\
\hline 26 & 14 & 15.75 & 11 \\
\hline 27 & 10 & 11.25 & 8 \\
\hline 28 & 9.2 & 10.35 & 7 \\
\hline 29 & 12.42 & 7.83 & 7 \\
\hline 30 & 14.26 & 8.99 & 8 \\
\hline 31 & 23 & 16.5 & 15 \\
\hline 32 & 6 & 6.75 & 3 \\
\hline 33 & 7.82 & 4.93 & 4 \\
\hline 34 & 9.52 & 10.54 & 6 \\
\hline 35 & 12.07 & 9.18 & 5 \\
\hline 36 & 6.8 & 7.65 & 7 \\
\hline 37 & 6.8 & 7.65 & 9 \\
\hline 38 & 11.5 & 12.25 & 12 \\
\hline 39 & 15.12 & 16.74 & 8 \\
\hline 40 & 14.91 & 15.34 & 15 \\
\hline 41 & 15.6 & 17.55 & 7 \\
\hline 42 & 7.2 & 8.1 & 6 \\
\hline 43 & 0.15 & 1 & 0 \\
\hline 44 & 0.2 & 1.6 & 0 \\
\hline 45 & 0.2 & 1.6 & 0 \\
\hline 46 & 0.15 & 1 & 0 \\
\hline
\end{tabular}




\begin{tabular}{|c|c|c|c|}
\hline 47 & 0.2 & 1.6 & 0 \\
\hline 48 & 0.2 & 1.6 & 0 \\
\hline 49 & 0.14 & 0.96 & 0 \\
\hline 50 & 0.058 & 0.4 & 0 \\
\hline 51 & 0.07 & 0.48 & 0 \\
\hline 52 & 0.058 & 0.4 & 0 \\
\hline 53 & 0.07 & 0.48 & 0 \\
\hline 54 & 0.058 & 0.4 & 0 \\
\hline 55 & 0.058 & 0.4 & 0 \\
\hline 56 & 0.058 & 0.4 & 0 \\
\hline 57 & 0.07 & 0.48 & 0 \\
\hline 58 & 0.118 & 0.82 & 0 \\
\hline 59 & 0.042 & 0.272 & 0 \\
\hline 60 & 0.042 & 0.272 & 0 \\
\hline 61 & 0.042 & 0.272 & 0 \\
\hline 62 & 0.042 & 0.272 & 0 \\
\hline
\end{tabular}

The pilot-slack bus is $68^{\text {th }}$ and voltage of which is 1.05 p.u.

Let's compare the initial powers with the optimum power in case of slight load refering to the table 8 as follows

Table 8. Comparison of generation

\begin{tabular}{|c|c|c|c|c|c|}
\hline \multirow[t]{2}{*}{$\begin{array}{l}\text { Bus } \\
\text { (i) }\end{array}$} & \multirow[t]{2}{*}{$\begin{array}{l}\text { Bus } \\
\text { Code }\end{array}$} & \multicolumn{2}{|c|}{$\begin{array}{c}\text { Initial } \\
\text { Generation }\end{array}$} & \multicolumn{2}{|c|}{$\begin{array}{l}\text { Optimum } \\
\text { Generation }\end{array}$} \\
\hline & & MW & MVAR & MW & MVAR \\
\hline 1 & Generator & 19 & -13.01599 & 18.8412 & -1.3805 \\
\hline 2 & Generator & 19 & -13.57139 & 18.9548 & -1.28669 \\
\hline 3 & Generator & 19 & -13.57139 & 18.9466 & -1.28665 \\
\hline 4 & Generator & 19 & -13.57139 & 18.9490 & -1.28686 \\
\hline 5 & Generator & 19 & -12.09338 & 18.9257 & -3.91745 \\
\hline 6 & Generator & 19 & -12.09338 & 18.9488 & -3.9178 \\
\hline 7 & Generator & 19 & -12.09338 & 18.9657 & -3.91777 \\
\hline 8 & Generator & 19 & -12.09338 & 18.9620 & -3.91775 \\
\hline 9 & Generator & 19 & -18.95797 & 19.3596 & -5.60036 \\
\hline 10 & Generator & 19 & -18.95797 & 19.3227 & -5.60023 \\
\hline 11 & Generator & 19 & -18.95797 & 19.3657 & -5.60036 \\
\hline 12 & Generator & 19 & -9.67552 & 19.1943 & -5.80648 \\
\hline 13 & Generator & 19 & -9.67552 & 19.2092 & -5.80652 \\
\hline 14 & Generator & 19 & -9.67552 & 19.1898 & -5.80646 \\
\hline 68 & Generator & 20.50082 & -9.67984 & 19.32799 & -11.02943 \\
\hline 63 & SVC & 0 & $\sigma$ & 0 & -2.71557 \\
\hline 64 & SVC & 0 & 0 & 0 & -25.9234 \\
\hline 65 & SVC & 0 & 0 & 0 & -32.3743 \\
\hline 66 & SVC & 0 & 0 & 0 & -39.7525 \\
\hline 67 & SVC & 0 & 0 & 0 & -25.2343 \\
\hline & ial Fuel & 20 & $.75 \$ / h$ & & \\
\hline ptin & num Fuel C & 20 & $3.71 \$ / \mathrm{h}$ & & \\
\hline & ving Fuel & & $3.04 \$ / \mathrm{h}$ & & \\
\hline
\end{tabular}

The comparison of bus voltages in case slight load is refering to the table 9 as follows 
TAP̈ CH PHAIUTTREÅIKHSON TAR̈ 16, SOÁ3- 20B

Table 9. Voltage comparison

\begin{tabular}{|c|c|c|}
\hline Bus (i) & $\begin{array}{c}\text { Initial Voltage } \\
\text { (p.u.) }\end{array}$ & $\begin{array}{c}\text { Optimum Voltage } \\
\text { (p.u.) }\end{array}$ \\
\hline 1 & 1.05 & 1.0666 \\
\hline 2 & 1.05 & 1.0679 \\
\hline 3 & 1.05 & 1.0679 \\
\hline 4 & 1.05 & 1.0679 \\
\hline 5 & 1.05 & 1.0613 \\
\hline 6 & 1.05 & 1.0613 \\
\hline 7 & 1.05 & 1.0613 \\
\hline 8 & 1.05 & 1.0613 \\
\hline 9 & 1.05 & 1.0672 \\
\hline 10 & 1.05 & 1.0672 \\
\hline 11 & 1.05 & 1.0672 \\
\hline 12 & 1.05 & 1.0604 \\
\hline 13 & 1.05 & 1.0604 \\
\hline 14 & 1.05 & 1.0604 \\
\hline 15 & 1.0753 & 1.0488 \\
\hline 16 & 1.0756 & 1.0517 \\
\hline 17 & 1.0457 & 1.0474 \\
\hline 18 & 1.0508 & 1.0526 \\
\hline 19 & 1.0633 & 1.065 \\
\hline 20 & 1.0539 & 1.0556 \\
\hline 21 & 1.0502 & 1.0519 \\
\hline 22 & 1.0535 & 1.0356 \\
\hline 23 & 1.0538 & 1.0359 \\
\hline 24 & 1.0294 & 1.0110 \\
\hline 25 & 1.0372 & 1.0189 \\
\hline 26 & 1.0589 & 1.0358 \\
\hline 27 & 1.0582 & 1.0351 \\
\hline 28 & 1.0626 & 1.0438 \\
\hline 29 & 1.0715 & 1.0529 \\
\hline 30 & 1.0704 & 1.0518 \\
\hline 31 & 1.0754 & 1.0571 \\
\hline 32 & 1.0554 & 1.0323 \\
\hline
\end{tabular}

\begin{tabular}{|c|c|c|}
\hline 33 & 1.0612 & 1.0323 \\
\hline 34 & 1.0528 & 1.0236 \\
\hline 35 & 1.0617 & 1.0329 \\
\hline 36 & 1.072 & 1.0454 \\
\hline 37 & 1.0783 & 1.0545 \\
\hline 38 & 1.0438 & 1.0455 \\
\hline 39 & 1.0312 & 1.0330 \\
\hline 40 & 1.0608 & 1.0625 \\
\hline 41 & 1.0397 & 1.0414 \\
\hline 42 & 1.0452 & 1.0469 \\
\hline 43 & 1.0738 & 1.0512 \\
\hline 44 & 1.0759 & 1.0696 \\
\hline 45 & 1.073 & 1.0681 \\
\hline 46 & 1.0784 & 1.0647 \\
\hline 47 & 1.0765 & 1.0744 \\
\hline 48 & 1.0682 & 1.0709 \\
\hline 49 & 1.0635 & 1.0459 \\
\hline 50 & 1.0573 & 1.0394 \\
\hline 51 & 1.0517 & 1.0337 \\
\hline 52 & 1.0538 & 1.0359 \\
\hline 53 & 1.0674 & 1.0445 \\
\hline 54 & 1.0658 & 1.0428 \\
\hline 55 & 1.0702 & 1.0516 \\
\hline 56 & 1.0745 & 1.056 \\
\hline 57 & 1.074 & 1.0555 \\
\hline 58 & 1.0788 & 1.0605 \\
\hline 59 & 1.0681 & 1.0453 \\
\hline 60 & 1.0656 & 1.0368 \\
\hline 61 & 1.0687 & 1.0400 \\
\hline 62 & 1.077 & 1.0487 \\
\hline 63 & 1.0633 & 1.0632 \\
\hline 64 & 1.0738 & 1.0251 \\
\hline 65 & 1.0635 & 1.0236 \\
\hline 66 & 1.0788 & 1.0335 \\
\hline 67 & 1.077 & 1.0232 \\
\hline 68 & 1.05 & 1.05 \\
\hline
\end{tabular}


The comparison of voltage levels may be graphically shown as refering to the figures 3 and 4.

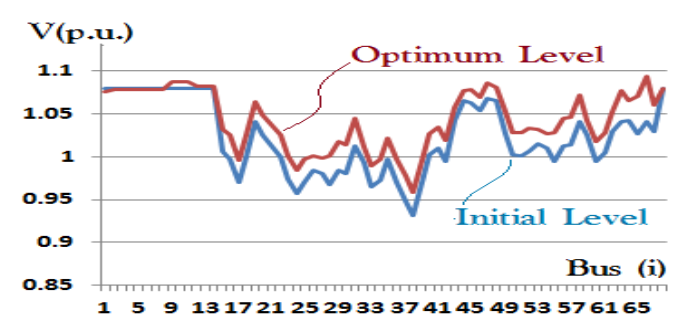

Fig.3 Voltage levels in case of loud load

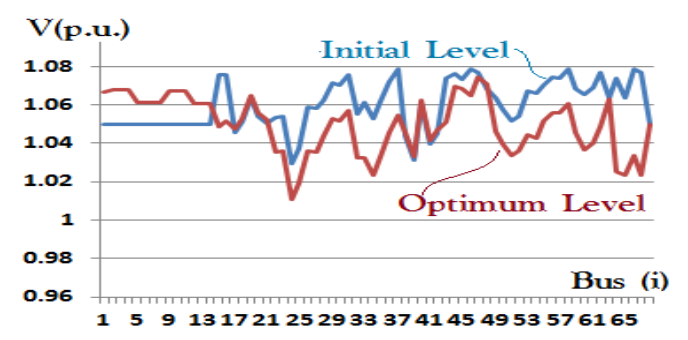

Fig.4 Voltage levels in case of slight load

\section{CONCLUSION}

The new algorithm of optimal pq_power flow problem is proved with good convergence.

The application of the speccific type fuel cost functions for the optimal pq_power generation problem allows to simulate the back-pressure effect of turbine regulation.

The process of calculation obtains a good results of voltage optimum levels according to the solution of optimal pq_power flow problem.

\section{Xét hiệu ứng áp suất ngược của tuabin các máy phát nhiệt điện nhằm tối ưu hóa công suất tác dụng và công suất phản kháng trong hệ thống điện}

- Lưu Hữu Vinh Quang

Trường Đại học Bách khoa, VNU-HCM

\section{TÓM TÁT:}

Đề xuất một giải thuật lập trình mới nhằm giải bài toán phân bố công suất và tối ưu hóa công suất $P(M W)$ và công suất $Q(M V A R)$ với các số liệu ví dụ kết quả tiêu biểu.
Đặc tính chi phí nhiên liệu của các máy phát nhiệt điện mà nhận được ở dạng hàm số đa thức bậc cao xếp chồng lên hàm số dạng sin có thể xét đến hiệu ứng áp suất ngược của turbin và giải quyết vấn đề tối ưu 
hóa công suất tác dụng của nhà máy nhiệt điện trong hệ thống điện.

Một công thức mới biểu thị tổng tổn hao công suất truyền tải là một hàm số bậc hai mà bao gồm một ma trận hệ số dạng vuông.

Từ khóa: Tối ưu hóa trạng thái xác lập; Hiệu ứng áp suất ngược của tuabin; Vận hành hệ thống.

\section{REFERENCES}

[1]. Luu Huu Vinh Quang. Considering the turbine back-pressure effect of thermal units for solving of generator pq_power optimization in power system. Authorship testimonial $\mathrm{N}^{\mathrm{0}} 3054 / 2013 / \mathrm{QTG}$ - Copyright Office of VN.

[2]. M.Sinkov, V.Vogoslovski, A.Kalinovski, A.Mozgovaya, A.Ogorodnikov. Optimization of Electric Power System Regimes. Kiev-High School Publisher (1973).

[3]. Luu Huu Vinh Quang. Using equivalent fuel cost characteristics of the thermal stations to optimize the p_power flows for economic power system operation. Proceedings of the $12^{\text {th }}$ conference on science and technology. ISEEE HCMUT 2011. Authorship testimonial $\mathrm{N}^{\circ} 2282 / 2013$ /QTG - Copyright Office of VN.
Công thức tính toán hệ số tổn hao này được đề xuất để giải quyết bài toán tối ưu hoá công suất phản kháng phát ra của các máy phát trong hệ thống điện có nhiều máy phát.
[4]. Luu Huu Vinh Quang. A methodology for solving of economical VAR compensation problem with voltage optimization gain considered in power system. (Autthorship testimonial $\mathrm{N}^{\mathrm{o}} 64$ - Copyright Office of VN -15-3-2001). Proceedings of the $8^{\text {th }}$ conference on science and technology (2526 April 2002). p.67-72. SEE and Power systems. VNU- HCM City University of Technology.

[5]. Luu Huu Vinh Quang. SVC scheduling according to p_power optimization with high order polynomial fuel cost functions fitting for generator steady-state stability. Proceedings of the $12^{\text {th }}$ conference on science and technology. ISEE HCMUT 2011, (Authorship testimonial $\mathrm{N}^{\mathrm{0}} 2972 / 2013 / \mathrm{QTG}$ - Copyright office of $\mathrm{VN})$. 